\title{
Análisis de la validez convergente de un instrumento informatizado para evaluar la atención en deportistas: Rejilla 1.0.
}

\section{Analysis of the convergent validity of a computerized instrument to evaluate attention in athletes: Reilla 1.0.}

\section{Análise da validade convergente de um instrumento informatizado para avaliar a atenção nos atletas: Rejilla 1.0.}

\author{
González-Guirval, F., Reigal, R. E., Morillo-Baro, J. P., Juárez-Ruiz de Mier, R., Hernández-Mendo, A., \\ y Morales-Sánchez, V. \\ Universidad de Málaga
}

\section{RESUMEN}

El objetivo de esta investigación fue analizar la validez convergente de un programa informatizado denominado Rejilla 1.0, que se construyó con el objetivo de evaluar la atención selectiva y para ser utilizado en contextos como el deporte. Un total de 99 participantes colaboraron en el estudio, con edades entre 19 y 36 años $(M \pm D T=25.15 \pm$ 3.56). Para valorar la validez convergente se realizaron análisis de correlaciones con otros instrumentos que se han utilizado tradicionalmente para evaluar la atención selectiva, como son el Test de Atención D2 y el Test ToulousePiéron. Los resultados encontrados pusieron de manifiesto correlaciones moderadas y bajas entre los instrumentos, lo cual sugiere relaciones entre ellos, aunque se deben tomar con cautela. Las medidas principales del Test de Atención D2 y el Test Toulouse-Piéron mostraron niveles moderados con los aciertos de Rejilla 1.0, pero las medidas secundarias manifestaron asociaciones bajas. Se sugiere que otras funciones cognitivas, además de atención selectiva, podrían participar en la realización de los ejercicios de Rejilla 1.0, lo cual se discute en el trabajo.

Palabras clave: atención; evaluación; informatizado.

\section{ABSTRACT}

The objective of this research was to analyze the convergent validity of a computerized program called Rejilla v.1, which was built with the aim of evaluating selective attention and to be used in contexts such as sport. A total of 99 participants collaborated on the study, aged between 19 and 36 years old $(M \pm S D=25.15 \pm 3.56)$. To assess convergent validity, correlation analyses were performed with other instruments that have traditionally been used to evaluate selective attention, such as the D2 Attention Test and the Toulouse-Piéron Test. The results found revealed moderate and low correlations between the instruments, suggesting relationships between them, although they should be taken with caution. The main measures of the D2 Test and the Toulouse-Piéron Test showed moderate levels with the hits of Rejilla v.1, but the secondary measures showed low associations. It is suggested that other cognitive functions, in addition to selective attention, could participate in the performance of Rejilla v.1 exercises, which is discussed at work.

Keywords: attention; evaluation; computerized.

\section{RESUMO}

Correspondence to: Rafael Reigal Garrido . Dirección Postal: Dpto.Campus de Teatinos, 29071. Universidad de Málaga. Málaga

(España). email: rafareigal@uma.es 


\section{González-Guirval, Reigal, Morillo-Baro, Juárez-Ruiz de Mier, Hernández-Mendo, \& Morales- Sánchez}

O objetivo desta pesquisa foi analisar a validade convergente de um programa informatizado chamado Rejilla 1.0, que foi construído com o objetivo de avaliar a atenção seletiva e ser utilizado em contextos como o esporte. Um total de 99 participantes colaboraram no estudo, variando na idade de 19 a 36 anos $(M \pm D P=25.15 \pm 3.56)$. Para avaliar a validade convergente, foram realizadas análises de correlação com outros instrumentos que tradicionalmente têm sido usados para avaliar a atenção seletiva, como o Teste de Atenção D2 e o Teste Toulouse-Piéron. Os resultados encontrados revelaram correlações moderadas e baixas entre os instrumentos, sugerindo relações entre eles, embora devam ser tomadas com cautela. As principais medidas do Teste D2 e do Teste toulouse-Piéron mostraram níveis moderados com os hits da Rejilla 1.0, mas as medidas secundárias mostraram associações baixas. Sugere-se que outras funções cognitivas, além da atenção seletiva, possam participar do desempenho dos exercícios Rejilla 1.0, que é discutido no trabalho.

Palavras chave: atenção; avaliação; computadorizado.

\section{INTRODUCCIÓN}

La atención es una capacidad cognitiva esencial para el funcionamiento del ser humano, la cual ha sido estudiada intensamente en las últimas décadas y cuyo interés sigue vigente (Atwi et al., 2018; Rosenberg et al., 2016). En ámbitos tan diversos como el educativo, laboral, o clínico la atención se ha mostrado relevante para adaptarse a las demandas de cada contexto y mostrar un mejor desempeño en un conjunto amplio de tareas (Durães, Carneiro, Bajo, y Novais, 2018; O'halloran et al., 2018; Rosenberg, Finn, Scatteheinost, Constable, y Chun, 2017). Específicamente, esta capacidad ha sido numerosas veces objeto de investigación en las ciencias del deporte, poniéndose de manifiesto su relevancia para el rendimiento competitivo en diferentes disciplinas y destrezas (Ducrocq, Wilson, Vine, y Derakshan, 2016; Love, Kannis-Dymand, y Lovell, 2018; Roca, Ford, y Memmert, 2018).

Es un constructo que puede tener diferentes manifestaciones como, entre otros, estado de alerta o arousal, atención selectiva, serial, dividida, alternante o sostenida (Posner y Petersen, 1990; Tamm, Epstein, Peugh, Nakonezny, y Hughes, 2013). En el contexto deportivo, la atención selectiva es una de sus dimensiones más explorada (Gonçalves et al., 2017; Harris, Wilson, y Vine, 2018; Memmert, 2009). Esta dimensión de la atención hace referencia a la habilidad que permite atender a una serie de estímulos, entre un conjunto presentado, mientras se ignoran a otros que no son relevantes (Giuliano, Karns, Neville, y Hillyard, 2014; Gomez-Ramirez, Hysaj, y Niebur, 2016). Es por ello que en estos contextos es una capacidad cognitiva que puede resultar fundamental, dado que facilita obtener información del entorno y evita la interferencia de otras fuentes distractoras que pudieran afectar al rendimiento deportivo (Romeas, Guldner, y Faubert, 2016; Wilson, Vine, y Wood, 2009). Por ello, en deportes colectivos como el fútbol la atención selectiva es crucial para una buena toma de decisión, pues permite seleccionar adecuadamente dónde se va a pasar y a quién, evitar elementos que pueden distraer como la acción del público o de los oponentes, así como decidir cuándo lanzar a portería (Romeas et al., 2016).

La atención, y en concreto la atención selectiva, es una capacidad cognitiva que puede ser evaluada y entrenada según se desprende de trabajos previos que así lo han constatado (Alves et al., 2014; Chun, Golomb y Turk-Browne, 2011; Romeas et al., 2016; Wass, Porayska-Pomsta y Johnson, 2011). Entre los instrumentos clásicos en papel útiles para su evaluación se pueden citar el test de atención D2 (Brickenkamp, 2001) o el Toulouse Piéron (Toulouse y Piéron, 1992). También existen software como el BrainTrain ${ }^{\circledR} \quad$ (www.braintrain.com) (Rabiner, Murray, Skinner, y Malone, 2010), Cognifit (www.cognifit.com) (Shatil, Mikulecka, Bellotti, y Bureš, 2014), Luminosity ${ }^{\circledR}$ (www.lumosity.com) (Morrison, Simone, Ng, y Hardy, 2015) o Brain Fitness (www.mindsparke.com) (Kletzel et al., 2016) que se pueden utilizar para esta finalidad, alcanzando algunos de ellos gran popularidad.

En el ámbito deportivo, también se han desarrollado específicamente dispositivos analógicos y digitales que permiten entrenar el funcionamiento cognitivo de los atletas, siendo útil para trabajar capacidades como la atención selectiva, dividida, sostenida, y otras habilidades superiores como las funciones ejecutivas. Entre ellos, se encuentran algunos como el Fitlight 


\section{Validez convergente Rejilla informática para deportistas}

Trainerтm, Dynavision D2тм, NeuroTracker, Eye tracking Sports, Vision Trainer ${ }^{\text {TM }}$ Senaptec Sensory Station o Sports Vision Performance de M\&S® (Appelbaum y Erickson, 2018; Schack, Bertollo, Koester, Maycock, y Essig, 2014; Romeas et al., 2016). El uso de software específico para evaluar estas destrezas en deportistas se ha extendido en los últimos años (Hernández-Mendo, Martínez-Jiménez, Pastrana-Brincones, y Morales-Sánchez, 2012; Hernández-Mendo, y Ramos-Pollán, 1995a, 1995b), y se han podido observar efectos positivos del entrenamiento mediante estos sistemas (Reigal et al., 2019).

La evaluación y el entrenamiento informatizado del funcionamiento cognitivo, como la atención, tiene una serie de ventajas respecto a otro tipo de medios. Por un lado, suelen ser dispositivos más versátiles, lo cual facilita adaptarlos al usuario (Kueider, Parisi, Gross, y Rebok, 2012), lo que puede favorecer su empleo en el contexto deportivo debido a las circunstancias de tiempo y espacio. Por otro lado, ejecuciones randomizadas facilitan la repetición de las pruebas y minimizan el efecto del aprendizaje, pudiendo efectuar un control más repetitivo de la evolución del deportista. Asimismo, los instrumentos informatizados incrementan la precisión de la medida, los tiempos de respuesta, de exposición al estímulo, etc. Por otro lado, poder modificar características visuales o sonoras de las tareas facilita mantener la motivación sobre el trabajo y ayudar a que los ejercicios se completen adecuadamente (Harris et al., 2018). Asimismo, el almacenamiento de datos grupales facilita el flujo de información entre los profesionales que trabajan con los deportistas, lo cual es esencial en estos contextos (González-Ruiz,
Domínguez-Alfonso, Chica-Merino, PastranaBrincones, y Hernández-Mendo, 2018).

Hernández-Mendo et al. (2012) desarrollaron un software denominado Rejilla 1.0 que actualmente está alojado en la plataforma de evaluación online MenPas (www.menpas.com) (González-Ruiz, HernándezMendo, y Pastrana-Brincones, 2010; González-Ruiz et al., 2018). Este programa permite evaluar fundamentalmente la atención selectiva, el cual se ha utilizado para evaluar y entrenar a deportistas (Reigal et al., , 2019). Básicamente se trata de una matriz de números (se pueden utilizar otros estímulos como letras, fotografías o símbolos), cuya tarea fundamental es identificar y tachar números (o los estímulos seleccionados). Para realizar esta tarea, se puede alterar aspectos como el color, el tiempo para realizar la tarea, el tamaño de la matriz, si se quiere que desaparezca el ítem cuando se tacha o si se quiere incluir un estímulo distractor (línea regulable en grosor y color, sonido o metrónomo). Evalúa esencialmente la atención selectiva porque hay que detectar entre un conjunto de ítems que son el objetivo, a la vez que se deben ignorar otros (Ver Figura 1)1. Por ello, el tipo de tarea es similar a otros instrumentos existentes en papel como son el test de atención D2 o el test Toulouse-Piéron (Brickenkamp, 2001; Toulouse y Piéron, 1992).

Con el propósito de explorar la validez de Rejilla 1.0 para ser utilizada en procesos de evaluación y entrenamiento de la atención selectiva de manera informatizada, el objetivo de este estudio fue analizar la validez convergente de la Rejilla 1.0 en una muestra de futbolistas, tomando como referencia el test de atención D2 y el test Toulouse-Piéron.
1 Para ver y consultar distintas opciones se ruega visitar https://www.youtube.com/watch? $\mathrm{v}=64 \mathrm{WSQhjyCvs \& list=}$ UUj2diXx fnk-2mIp BY8BYA\&index $=15 \mathrm{y}$ https://www.youtube.com/watch? $\mathrm{v}=\mathrm{cFLy}$ oDUQx $8 \&$ list $=$ UUj2djXx_fnk-2mIp_BY8BYA\&index $=16$ 


\section{González-Guirval, Reigal, Morillo-Baro, Juárez-Ruiz de Mier, Hernández-Mendo, \& Morales- Sánchez}

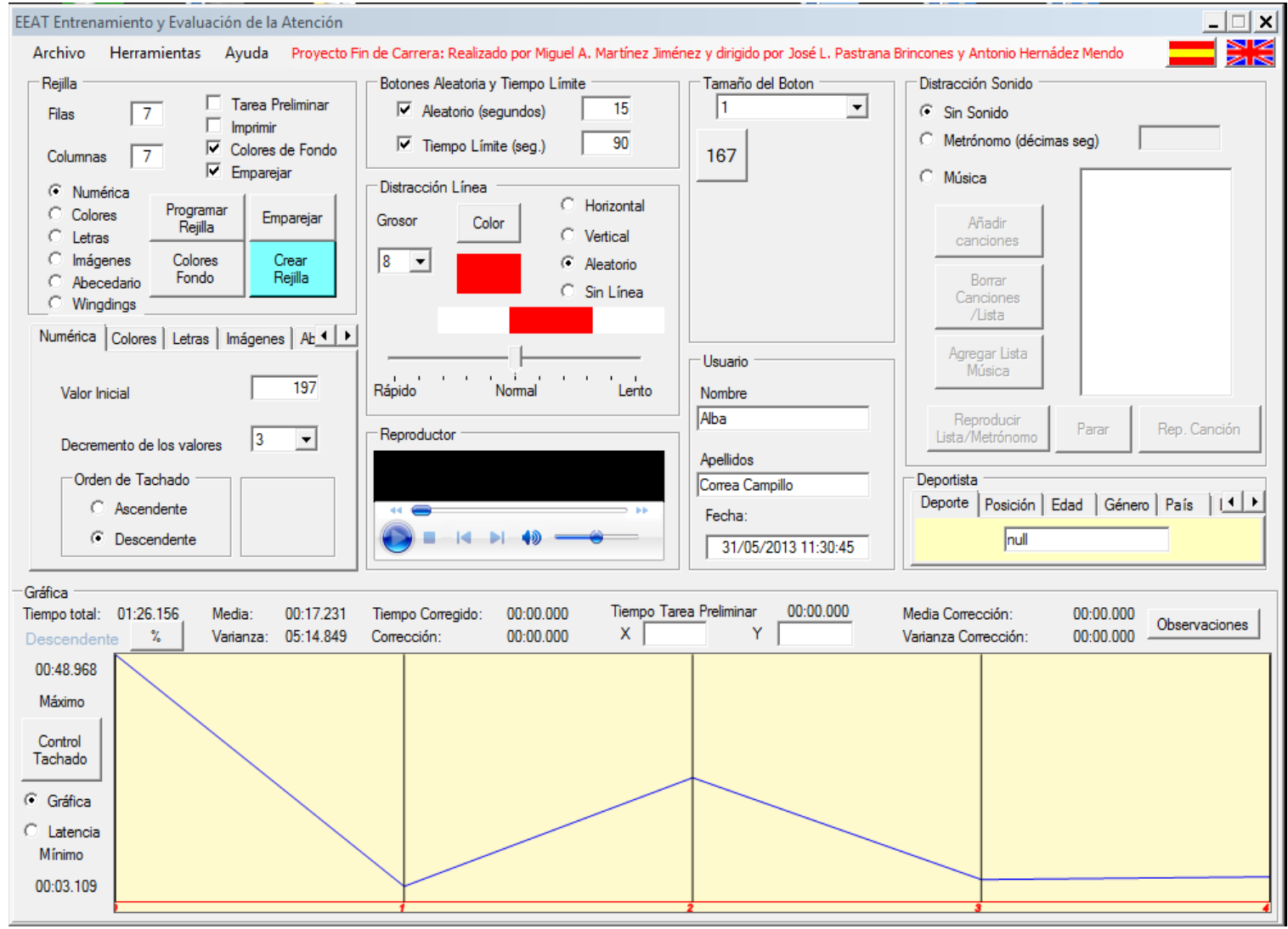

Figura 1. Captura de pantalla de Rejilla v.1. con sus diferentes opciones.

\section{MATERIAL Y MÉTODOS}

\section{Participantes}

En esta investigación participaron 99 futbolistas de la ciudad de Málaga, con edades entre 19 y 36 años $(M \pm$ $D T=25.15 \pm 3.56)$. La muestra se obtuvo en diferentes instituciones educativas de la ciudad de Málaga: Universidad de Málaga (España), EADE Estudios Universitarios (Málaga, España) y Escuela de Entrenadores (CEDIFA, sede de Málaga, España). El $15.15 \%(n=15)$ fue de género femenino y el $84.85 \%$ $(n=84)$ fue de género masculino. La muestra fue seleccionada de manera incidental en diferentes organizaciones y todos eran deportivas con más de 10 años de experiencia en práctica deportiva. Se consideró como criterio de exclusión aquellos que manifestaran algún tipo de dificultad psicológica o física que le impidiera realizar adecuadamente las pruebas de esta investigación. Asimismo, se excluyeron a los participantes que no cumplimentaran adecuadamente las tareas. De los 102 iniciales se descartaron a 3 por este último motivo.

\section{Instrumentos}

a) Test de Rejilla 1.0 (Hernández-Mendo et al., 2012). Este programa se trata de una aplicación para Windows desarrollada bajo la plataforma .NET en lenguaje de programación C\# y con el entorno de programación Visual Studio. Con este software se pueden realizar diferentes ejercicios basados fundamentalmente en la localización de estímulos en una rejilla regulable en tamaño. Estos estímulos pueden ser números, colores, letras, imágenes, abecedario y windings. Se puede programar el tamaño de los ítems, el color de fondo, que cambien de lugar, el tiempo de presentación o la aparición de líneas distractoras. Permite conocer el tiempo entre cada estímulo y los tiempos de latencia. Calcula indicadores de eficacia y de efectividad, así como porcentajes de aciertos/errores- relativos/absolutos. Aunque según la configuración del ejercicio se pueden entrenar y evaluar diferentes tipos de atención, 


\section{Validez convergente Rejilla informática para deportistas}

fundamentalmente este programa se centra en la Atención Selectiva o Focal/Atención Serial (EstévezGonzález, García-Sánchez, y Junqué, , 1997).

b) Test de Atención d2 (Brickenkamp y Zillmer, 2002). Este test se emplea para analizar la capacidad para atender estímulos relevantes de forma rápida $\mathrm{y}$ precisa, ignorando los irrelevantes, lo cual se considera una manifestación de la atención selectiva y la concentración. En este test hay que discriminar entre 47 caracteres en 14 filas, con un total de 658 elementos. Se dispone de 20 segundos para realizar cada fila. Los estímulos contienen las letras "d" o "p", que pueden estar acompañadas de una o dos rayas en la parte superior del ítem, en la parte inferior o en ambas. Se deben tachar las "d" con 2 rayas (independientemente de la posición). Siempre se completa el test de izquierda a derecha y de arriba hacia abajo. Las puntuaciones que se obtienen son: TR (elementos procesados), TA (aciertos), $\mathrm{O}$ (omisiones), $\mathrm{C}$ (comisiones o errores), TOT [efectividad en la tarea $=\mathrm{TR}-(\mathrm{O}+\mathrm{C})], \mathrm{CON}$ (concentración $=\mathrm{TA}-\mathrm{C}$ ), $\mathrm{TR}+$ (último estímulo analizado en la fila con más elementos intentados), TR- (último estímulo analizado en la fila con menos elementos intentados) y VAR [índice de variación entre el último estímulo analizado entre distintas filas $=(\mathrm{TR}+)-(\mathrm{TR}-)]$. Esta prueba posee una fiabilidad test-retest en el estudio original superior a .90 .

c) Test Toulouse-Piéron (Toulouse y Piéron, 1992). Este test se emplea para analizar la capacidad para atender estímulos relevantes, lo cual se considera una manifestación de la atención selectiva y la concentración. Al realizarse la tarea durante 10 minutos, es un instrumento adecuado para evaluar la resistencia a la fatiga. En este test hay que discriminar en una matriz de 1600 ítems dos tipos que se presentan como modelo. Los estímulos son cuadrados pequeños con una línea que puede situarse en posición horizontal, vertical u oblicua, adherida a cualquier posición del perímetro del cuadrado. Para la realización del test se dispone de 10 minutos. Las puntuaciones que se obtienen son: $\mathrm{P}$ (procesados), $\mathrm{R}$ (respuestas), A (aciertos), O (omisiones), E (errores), $\mathrm{PD}$ [puntuación directa $=\mathrm{A}-(\mathrm{E}+\mathrm{O})]$ y $\mathrm{E}$ (eneatipos).

\section{Procedimiento}

La muestra se obtuvo en diferentes instituciones de la ciudad de Málaga (España): Universidad de Málaga,
EADE Málaga y Escuela de Entrenadores (CEDIFA). Se contactó con la dirección de cada institución o los coordinadores de la sección correspondiente, para solicitar permiso para realizar la investigación. Tras ello, se contactó con los alumnos/as, se les explicó el propósito del estudio y se les indicó que la participación sería voluntaria, anónima y los datos se utilizarían únicamente con fines de investigación. Asimismo, se les informó que en cualquier momento podrían dejar de formar parte de la investigación. Tras ello, se obtuvo el pertinente consentimiento informado. Además, durante el proceso de investigación se respetaron los principios éticos de la declaración de Helsinki (World Medical Association, 2013).

Las evaluaciones se realizaron en un único día durante 60 minutos aproximadamente. En primer lugar, se realizaron el test de atención D2 y el test de ToulousePiéron. Para ello, se explicó el protocolo de las diferentes pruebas y se resolvieron las dudas que pudieran surgir. En segundo lugar, se realizaron las diferentes pruebas seleccionadas de Rejilla 1.0. Específicamente se realizaron cuatro ejercicios en una matriz numérica de $7 \times 7$, todos con una duración máxima de 90 segundos: (1) sin emparejar (sin marcar un número en la matriz principal que aparece indicado fuera de esa matriz), ascendente, tachado del número marcado, cambio de posición de los números de la matriz cada 15 segundos y con línea distractora; (2) emparejado (marcando un número en la matriz principal que aparece indicado fuera de esa matriz), tachado del número marcado, aleatorio (según número indicado fuera de la matriz) y con línea distractora; (3) sin emparejar, ascendente, sin tachado del número marcado, cambio de la posición de los números cada 15 segundos y con línea distractora; (4) emparejado, sin tachado del número marcado, aleatorio (según número marcado fuera de la matriz) y con línea distractora.

\section{Análisis estadístico}

Se efectuaron análisis descriptivos e inferenciales. Se comprobó la normalidad de los datos con la prueba de Kolmogorov-Smirnov. Se efectuaron análisis de correlaciones con la prueba de Pearson y Spearman. Para procesar los datos se utilizó el paquete de análisis estadístico IBM SPSS Statistics 24.0. 


\section{González-Guirval, Reigal, Morillo-Baro, Juárez-Ruiz de Mier, Hernández-Mendo, \& Morales- Sánchez}

\section{RESULTADOS}

En la Tabla 1 se muestran los estadísticos descriptivos y de normalidad de las variables objeto de estudio.
Como se puede observar, algunas de las medidas analizadas mostraron problemas de normalidad.

Tabla 1

Medidas descriptivas y prueba de Kolmogorov-Smirnov para las variables analizadas

\begin{tabular}{|c|c|c|c|c|c|}
\hline & $M$ & $D T$ & $A$ & $K$ & $K-S$ \\
\hline \multicolumn{6}{|l|}{ Test D2 } \\
\hline D2_TR & 68.52 & 18.00 & .17 & -.79 & 1.11 \\
\hline D2_TA & 62.99 & 18.89 & .06 & -.37 & 1.15 \\
\hline D2_O & 39.82 & 19.44 & -.12 & -.61 & .71 \\
\hline D2_C & 46.68 & 14.27 & -.57 & -.86 & $2.98 * * *$ \\
\hline D2_TOT & 66.64 & 18.33 & .04 & -.39 & 1.16 \\
\hline $\mathrm{D} 2 \_\mathrm{CON}$ & 63.18 & 19.04 & .06 & -.49 & 1.12 \\
\hline D2_TR+ & 65.89 & 12.05 & -1.13 & .60 & $2.53 * * *$ \\
\hline D2_TR- & 69.77 & 18.89 & .00 & -1.07 & $1.37 *$ \\
\hline D2_VAR & 53.00 & 22.10 & -.69 & .08 & 1.03 \\
\hline \multicolumn{6}{|l|}{ TP } \\
\hline TP_R & 240.26 & 49.87 & .30 & -.38 & .46 \\
\hline TP_O & 32.11 & 21.81 & .87 & .42 & 1.22 \\
\hline TP_P & 271.77 & 59.24 & .39 & -.49 & .81 \\
\hline TP_A & 239.67 & 49.89 & .30 & -.38 & .50 \\
\hline TP_E & .60 & 1.14 & 2.57 & 7.30 & $2.74 * * *$ \\
\hline TP_ENE & 5.06 & 1.75 & .29 & -.53 & $1.81 * *$ \\
\hline \multicolumn{6}{|l|}{ Rejilla 1.0} \\
\hline E1_Ac & 16.59 & 8.89 & 1.25 & 2.20 & 1.13 \\
\hline E2_Ac & 10.06 & 4.64 & .62 & .00 & 1.25 \\
\hline E3_Ac & 11.13 & 5.70 & .52 & .48 & .85 \\
\hline E4_Ac & 9.18 & 4.08 & .11 & -.51 & .73 \\
\hline E1_Er & 1.73 & 3.15 & 4.37 & 25.97 & $3.12 * * *$ \\
\hline E2_Er & 2.89 & 3.41 & 3.48 & 15.71 & $2.71 * * *$ \\
\hline E3_Er & 4.98 & 7.20 & 2.63 & 8.37 & $2.43 * * *$ \\
\hline E4_Er & 3.30 & 4.43 & 2.93 & 9.12 & $3.05 * * *$ \\
\hline
\end{tabular}

Nota. A= Asimetría; K= Curtosis; K-S= Prueba de Kolmogórov-Smirnov; D2 = Test D2; D2_TR = Total número de intentos; D2_TA = Total de aciertos; D2_O = Omisiones; D2_C = Comisiones; D2_TOT = Efectividad total en la prueba; D2_CON = Índice de concentración; D2_VAR = Índice de variación; D2_TR+ = Línea con mayor número de elementos intentados; D2_TR- = Línea con menor número de elementos intentados; TP = Toulouse-Piéron; TP_P = Procesados); TP_R = Respuestas; TP_A = Aciertos; TP_O = omisiones; TP_E = Errores; TP_E $=$ Eneatipos; E1 - E4 = Ejercicios del 1 al 4; Ac = Aciertos; Er = Errores.

Los análisis de correlaciones (Tabla 2) mostraron relaciones significativas entre las variables analizadas. Destacan las relaciones moderadas, o próximas a ellas, entre las medidas principales del test de atención D2 y del Toulouse-Piéron con las puntuaciones de aciertos en los cuatro ejercicios de Rejilla 1.0 ( $r=.30$ a .47). Sin embargo, apenas se generaron relaciones con los errores de Rejilla 1.0, sólo algunas con las puntuaciones de comisiones del test de atención D2. Respecto a las respuestas dadas y aciertos del test de atención D2 y del Toulouse-Piéron, las relaciones con los aciertos de los ejercicios de Rejilla 1.0 tuvieron un nivel bajo o moderado $(r=.22$ a .42$)$. 


\section{Validez convergente Rejilla informática para deportistas}

Tabla 2

Análisis de correlaciones entre las variables del estudio

\begin{tabular}{|c|c|c|c|c|c|c|c|c|}
\hline & \multicolumn{4}{|c|}{ Aciertos (Rejilla 1.0) } & \multicolumn{4}{|c|}{ Errores (Rejilla 1.0) } \\
\hline & E1 & E2 & E3 & E4 & E1 & E2 & E3 & $\mathrm{E} 4$ \\
\hline \multicolumn{9}{|l|}{ D2 } \\
\hline D2_TR & $.22 *$ & $.27 * *$ & $.27 * *$ & $.26 * *$ & .08 & .01 & -.09 & .01 \\
\hline D2_TA & $.24 *$ & $.23^{*}$ & $.33 * * *$ & $.34 * * *$ & -.04 & .08 & -.01 & -.04 \\
\hline D2_O & .12 & .13 & $.21 *$ & $.20^{*}$ & $-.25^{*}$ & .07 & .01 & -.06 \\
\hline D2_C & .01 & $.21 *$ & .11 & .06 & -.17 & $-.30 * *$ & $-.20 *$ & $-.26 * *$ \\
\hline D2_TOT & $.41 * * *$ & $.34 * * *$ & $.36 * * *$ & $.39 * * *$ & -.06 & .06 & -.03 & -.07 \\
\hline D2_CON & $.37 * * *$ & $.30 * *$ & $.37 * * *$ & $.39 * * *$ & -.11 & .09 & .01 & -.15 \\
\hline D2_TR+ & .17 & $.18 \mathrm{a}$ & .17 & $.29 * *$ & .05 & -.04 & .03 & -.02 \\
\hline D2_TR- & $.23 *$ & $.24 *$ & $.31 * *$ & $.23^{*}$ & .03 & .02 & -.17 & -.10 \\
\hline D2_VAR & .04 & .05 & $.20 *$ & .08 & .08 & -.03 & .14 & .15 \\
\hline \multicolumn{9}{|l|}{$\mathrm{TP}$} \\
\hline TP_R & $.42 * * *$ & $.27 * *$ & $.24 *$ & $.29 * *$ & -.02 & -.04 & .05 & -.03 \\
\hline TP_O & .12 & -.01 & .06 & -.05 & .08 & -.08 & -.16 & -.11 \\
\hline TP_P & $.40 * * *$ & $.23^{*}$ & $.22 *$ & $.23 *$ & .07 & -.06 & -.02 & -.07 \\
\hline TP_A & $.42 * * *$ & $.28 * *$ & $.24 *$ & $.29 * *$ & -.02 & -.04 & .05 & -.04 \\
\hline TP_E & -.05 & -.15 & -.10 & $-.22 *$ & .17 & .06 & -.12 & .16 \\
\hline TP_ENE & $.47 * * *$ & $.36 * * *$ & $.30 * *$ & $.37 * * *$ & -.10 & -.02 & .10 & -.02 \\
\hline
\end{tabular}

Nota. D2 = Test D2; D2_TR = Total número de intentos; D2_TA = Total de aciertos; D2_O = Omisiones; D2_C = Comisiones $;$ D2_TOT = Efectividad total en la prueba; D2_CON = Índice de concentración; D2_VAR = Índice de variación; D2_TR+ = Línea con mayor número de elementos intentados; D2_TR- = Línea con menor número de elementos intentados; TP = Toulouse-Piéron; TP_P $=$ Procesados); TP_R = Respuestas; TP_A = Aciertos; TP_O = omisiones; TP_E = Errores; TP_E = Eneatipos; E1 - E4 = Ejercicios del 1 al 4.

Asimismo, las relaciones entre las principales puntuaciones del test D2 (TOT y CON) mostraron correlaciones moderadas con los eneatipos del Toulouse-Piéron $(r=.42$ a .47) (tabla 3$)$.

Tabla 3

Análisis de correlaciones entre las variables del estudio

\begin{tabular}{|c|c|c|c|c|c|c|}
\hline & \multicolumn{6}{|c|}{ TP } \\
\hline & $\mathrm{R}$ & $\mathrm{O}$ & $\mathrm{P}$ & $\mathrm{A}$ & $\mathrm{E}$ & ENE \\
\hline \multicolumn{7}{|l|}{ Test D2 } \\
\hline D2_TR & $.42 * * *$ & .18 & $.41 * * *$ & $.42 * * *$ & .03 & $.37 * * *$ \\
\hline D2_TA & $.40 * * *$ & -.06 & $.32 * *$ & $.41 * * *$ & -.13 & $.46 * * *$ \\
\hline D2_O & .06 & $-.38 * * *$ & -.08 & .07 & $-.22 *$ & $.22 *$ \\
\hline D2_C & -.05 & -.10 & -.07 & -.04 & $-.34 * * *$ & .01 \\
\hline D2_TOT & $.47 * * *$ & .11 & $.44 * * *$ & $.47 * * *$ & -.06 & $.46^{* * * *}$ \\
\hline D2_CON & $.42 * * *$ & -.01 & $.35 * * *$ & $.42 * * *$ & -.14 & $.45^{* * * *}$ \\
\hline D2_TR+ & $.30 * *$ & $.22 *$ & $.33 * * *$ & $.30 * *$ & .01 & $.21 *$ \\
\hline D2_TR- & $.30^{*}$ & .15 & $.30 * *$ & $.29 * *$ & -.05 & $.26 *$ \\
\hline D2_VAR & $.25^{*}$ & .06 & .19 & $.26^{*}$ & .07 & $.22 *$ \\
\hline
\end{tabular}

Nota. D2 = Test D2; D2_TR = Total número de intentos; D2_TA = Total de aciertos; D2_O = Omisiones; D2_C = Comisiones; D2_TOT = Efectividad total en la prueba; D2_CON = Índice de concentración; D2_VAR = Índice de variación; D2_TR+ = Línea con mayor número de elementos intentados; D2_TR- = Línea con menor número de elementos intentados; $\mathrm{TP}=$ Toulouse-Piéron; $\mathrm{TP} \_\mathrm{P}=$ Procesados); $\mathrm{TP} \_\mathrm{R}=$ Respuestas; TP_A = Aciertos; TP_O = omisiones; TP_E = Errores; TP_E = Eneatipos. 


\section{González-Guirval, Reigal, Morillo-Baro, Juárez-Ruiz de Mier, Hernández-Mendo, \& Morales- Sánchez}

\section{DISCUSIÓN}

El objetivo de esta investigación fue evaluar la validez convergente de la Rejilla 1.0, utilizando como criterio las puntuaciones de atención y concentración de los test de atención D2 y Toulouse-Piéron, los cuales se han utilizado ampliamente en investigaciones previas para evaluar la atención selectiva y la concentración (Gall et al., 2018; Walsh et al., 2018). En general, se observan relaciones bajas y moderadas entre las puntuaciones de los instrumentos analizados, y específicamente relaciones moderadas entre las principales puntuaciones de los tres instrumentos. Estos resultados satisfacen parcialmente el objetivo del estudio. Aunque en términos generales se ponen de relieve vínculos entre los tests, la fuerza moderada de la relación requiere posteriores estudios que confirmen y clarifiquen los vínculos entre ellos. En cualquier caso, los resultados encontrados sugieren que Rejilla 1.0 puede ser un instrumento útil para analizar el funcionamiento cognitivo $\mathrm{y}$, específicamente la atención selectiva, si bien los ejercicios configurados en este trabajo podrían requerir el uso de otras destrezas cognitivas que también pudieran estar implicadas en los resultados encontrados.

En primer lugar, hay que destacar la existencia de correlaciones entre las puntuaciones principales del test D2 (D2) y Toulouse-Piéron (TP) con los aciertos de los ejercicios de Rejilla 1.0. No sólo las relativas a las puntuaciones principales D2 y TP, sino también algunas parciales como elementos procesados 0 aciertos. Esto sugiere que las capacidades cognitivas fundamentales implicadas durante la ejecución de los diferentes ejercicios podrían ser similares. De hecho, tanto el test de atención D2 como el test de Toulouse Piéron analizan fundamentalmente la atención selectiva y la concentración, y la Rejilla 1.0 se construyó con el objetivo de evaluar esas habilidades con tareas parecidas. Así, en los ejercicios de los tres instrumentos se demanda atender a una serie de elementos e ignorar otros, lo cual determinaría el éxito en la tarea realizada y es la habilidad fundamental que se requiere en las tareas de atención selectiva (Giuliano et al., 2014). Asimismo, las tareas requeridas en los test de atención D2 y ToulousePiéron requieren mantener dicha atención durante un periodo de tiempo, lo cual estaría determinado por la capacidad de concentración de los participantes durante los ejercicios (Blotenberg y Schmidt-Atzert, 2019).

Sin embargo, las puntuaciones de las pruebas de correlaciones han sido bajas o moderadas, lo cual mostraría una validez convergente limitada. Esto podría deberse a varios motivos. En primer lugar, el tiempo de trabajo en cada test ha sido diferente, como ya se ha comentado. Mientras en Rejilla 1.0 se ha trabajado durante 90 segundos, el test D2 ha durado 280 segundos y el Toulouse-Piéron 600 segundos. Este es uno de los aspectos que se debería atender en futuros trabajos, dado que estos requerimientos podrían estar afectando a los resultados. En segundo lugar, las características de los ejercicios realizados en Rejilla 1.0 quizás están dotados de elementos que impliquen a otras habilidades. Por ejemplo, que los ítems sean dinámicos, que cambien de posición o la aparición de una línea distractora podría estar requiriendo otras capacidades más complejas como memoria de trabajo, flexibilidad cognitiva o control inhibitorio (Stuss, 2011; Zaehringer, Falquez, Schubert, Nees, y Barnow, 2018). Aunque es cierto que para realizar D2 y TP hace falta memorizar una serie de ítems objetivos, en algunos ejercicios de rejilla hay que memorizar de forma dinámica el elemento tachado. También, en Rejilla 1.0 la matriz de datos cambia la posición de los números, con lo que hay que ser flexible a la hora de establecer una estrategia de tachado. Asimismo, a estas habilidades, habría que añadir cierta capacidad para inhibir el tachado cuando en la tarea el número anterior marcado no desaparece de la matriz. Por ello, estas diferencias podrían estar requiriendo un desarrollo de un funcionamiento más complejo para completar la tarea.

Sí se considera consistente el patrón encontrado en las relaciones entre los diferentes tests. Aunque algunas correlaciones son bajas y otras moderadas, el estudio de las relaciones entre el D2 y el TP han mostrado puntuaciones similares, siendo bajas o moderadas las relaciones entre ellas y siendo más visibles las asociaciones entre puntuaciones principales, así como elementos procesados o aciertos, que con otras puntaciones como errores o comisiones. Por ello, se puede considerar que existe coherencia en los valores encontrados y, aunque se esperaba encontrar vínculos más fuertes entre las puntuaciones, se podría considerar aceptable los resultados encontrados y sugerir que los ejercicios de Rejilla 1.0 estarían 


\section{Validez convergente Rejilla informática para deportistas}

evaluando atención selectiva, al menos que fuera una de las capacidades cognitivas principales implicada en la tarea.

La principal limitación de este trabajo sería el requerimiento de otras pruebas de evaluación cognitiva necesarias para descartar o asumir otro tipo de relaciones y valorar si Rejilla 1.0 estaría evaluando también otro tipo de capacidad. Además, podría existir interferencias debido al formato de la prueba (lápiz y papel vs. informatizada). Por ello, sería interesante analizar la validez convergente de la Rejilla 1.0 con otros instrumentos de evaluación cognitiva que ofrezcan más variables mediante las cuales se puedan contrastar los resultados, así como utilizar otras pruebas informatizadas que permitan analizar las diferencias entre ambos tipos de ejecución. Asimismo, el orden en la realización de las pruebas podría ser un factor limitante en los resultados obtenidos. Por ello, sería adecuado en próximos trabajos efectuar balanceos en el orden de las pruebas para comprobar si los resultados se mantienen. Otra alternativa podría ser evaluar cada instrumento en días diferentes, aunque para ello habría que controlar otras posibles fuentes de distorsión como el horario de realización, fatiga acumulada a lo largo del día o descanso previo. Por último, el presente estudio ha utilizado una muestra mayoritariamente de género masculino, por lo que se podría analizar en próximas investigaciones si el género es una variable que pudiera condicionar los resultados. A pesar de dichas limitaciones, la coherencia de las asociaciones encontradas entre las puntuaciones permite sugerir que Rejilla 1.0 estaría evaluando atención selectiva, lo cual es asumible en función del tipo de ejercicio empleado (matrices de tachado), el cual ha sido una tarea clásica para la valoración de esta capacidad.

Por ello, a pesar de ser necesario analizar las relaciones con otras capacidades cognitivas, se puede considerar que Rejilla 1.0 es una buena herramienta para la evaluación cognitiva, analizando fundamentalmente atención selectiva, y que podría ser empleada en diferente tipo de población. Específicamente en deportistas, este tipo de software es interesante por su versatilidad, por la capacidad de generar tareas atractivas para la población participante y por su capacidad para generar datos que pudieran ser utilizados posteriormente en el análisis y mejorar de la capacidad cognitiva en este tipo de muestras. Una de las posibilidades es que Rejilla 1.0 pudiera ser un instrumento que permitiera evaluar un amplio rango de variables cognitivas mediante la manipulación de sus diferentes modos de ejercicios. No sólo otros tipos de atención sino capacidades cognitivas más complejas como las funciones ejecutivas, específicamente aspectos como la memoria de trabajo, flexibilidad cognitiva o control inhibitorio. De eso modo, podría ser utilizada en múltiples contextos y objetivos, y se trataría de una herramienta muy versátil para ser utilizada tanto para evaluación como entrenamiento cognitivo.

\section{REFERENCIAS}

1. Alves, C. R., Tessaro, V. H., Teixeira, L. A., Murakava, K., Roschel, H., Gualano, B., y Takito, M. Y. (2014). Influence of acute high-intensity aerobic interval exercise bout on selective attention and short-term memory tasks. Perceptual and Motor Skills, 118(1), 63-72. https://doi.org/10.2466/22.06.PMS.118k10w4

2. Appelbaum, L. G., y Erickson, G. (2018). Sports vision training: A review of the state-of-the-art in digital training techniques. International Review of Sport and Exercise Psychology, 11(1), 160-189. https://doi.org/10.1080/1750984X.2016.1266376

3. Atwi, S., Metcalfe, A. W. S., Robertson, A. D., Rezmovitz, J., Anderson, N. D., y MacIntosh, B. J. (2018). Attention-Related Brain Activation Is Altered in Older Adults With White Matter Hyperintensities Using Multi-Echo fMRI. Frontiers in Neuroscience, 12, 748. https://doi.org/10.3389/fnins.2018.00748

4. Blotenberg, I., and Schmidt-Atzert, L. (2019). On the Characteristics of Sustained Attention Test Performance. European Journal of Psychological Assessment. 5759/a000543

5. Brickenkamp, R. (2001). D-2. Attention task. Madrid: TEA ediciones.

6. Brickenkamp, R., y Zillmer, E. (2002). Test de atención. Madrid: TEA ediciones.

7. Chun, M.M., Golomb, J.D. y Turk-Browne, N.B. (2011). A taxonomy of external and internal attention. Annual Reviews of Psychology, 62, 73101. 


\section{González-Guirval, Reigal, Morillo-Baro, Juárez-Ruiz de Mier, Hernández-Mendo, \& Morales- Sánchez}

https://doi.org/10.1146/annurev.psych.093008.10 0427

8. Ducrocq, E., Wilson, M., Vine, S., y Derakshan, N. (2016). Training attentional control improves cognitive and motor task performance. Journal of Sport and Exercise Psychology. 38, 521-533. https://doi.org/10.1123/jsep.2016-0052

9. Durães, D., Carneiro, D., Bajo, J., y Novais, P. (2018). Modelling a smart environment for nonintrusive analysis of attention in the workplace. Expert Systems, 35(5), e12275. https://doi.org/10.1111/exsy.12275

10. Estévez-González, A., García-Sánchez, C. y Junqué, C. (1997). La atención: una compleja función cerebral. Revista de Neurología, 25(148), 1989-1997.

11. Gall, S., Adams, L., Joubert, N., Ludyga, S., Müller, I., Nqweniso, S., ... y Utzinger, J. (2018). Effect of a 20-week physical activity intervention on selective attention and academic performance in children living in disadvantaged neighborhoods: A cluster randomized control trial. PloS One, 13(11), e0206908. https://doi.org/10.1371/journal.pone.0206908

12. Giuliano, R. J., Karns, C. M., Neville, H. J., y Hillyard, S. A. (2014). Early auditory evoked potential is modulated by selective attention and related to individual differences in visual working memory capacity. Journal of Cognitive Neuroscience, 26, 2682-2690. https://doi.org/10.1162/jocn_a_00684

13. Gomez-Ramirez, M., Hysaj, K., y Niebur, E. (2016). Neural mechanisms of selective attention in the somatosensory system. Journal of Neurophysiology, 116(3), 1218-1231. https://doi.org/10.1152/jn.00637.2015

14. Gonçalves, E., Noce, F., Barbosa, M. A. M., Figueiredo, A. J., Hackfort, D., y Teoldo, I. (2017). Correlation of the peripheral perception with the maturation and the effect of the peripheral perception on the tactical behaviour of soccer players. International Journal of Sport and Exercise Psychology, 1-13. http://doi.org/10.1080/1612197X.2017.1329222

15. González-Ruiz, S. L., Domínguez-Alfonso, R., Chica-Merino, E., Pastrana-Brincones, J. L., and
Hernández-Mendo, A. (2018). A virtual platform for on-line evaluation and research: MenPas. Cuadernos de Psicología del Deporte, 18(3), 2648.

16. González-Ruiz, S.L., Hernández-Mendo, A., Pastrana-Brincones, J.L. (2010). Herramienta software para la evaluación psicosocial de deportistas y entornos deportivos. Lecturas: EF y Deportes. Revista Digital, 15(144), mayo. http://www.efdeportes.com/efd144/evaluacionpsicosocial-de-deportistas.htm

17. Harris, D. J., Wilson, M. R., y Vine, S. J. (2018). A systematic review of commercial cognitive training devices: implications for use in sport. Frontiers in Psychology, 9, 709. https://doi.org/10.3389/fpsyg.2018.00709

18. Hernández-Mendo, A., Martínez-Jiménez, M. A, Pastrana-Brincones, J. L. y Morales-Sánchez, V. (2012). Programa informático para evaluación y entrenamiento de la atención. Revista Iberoamericana de Psicología del Ejercicio y el Deporte, 7(2), 339-358.

19. Hernández-Mendo, A. y Ramos-Pollán, R. (1995a). Tarea informática para la evaluación y entretenimiento de la atención: aplicación en el entrenamiento deportivo. Anales de Psicología, 11(2), 183-192.

20. Hernández-Mendo, A. y Ramos-Pollán, R. (1995b). Aplicación informática para evaluación y entrenamiento de la atención en psicologia del deporte. Psicothema, 7(3), 527-529.

21. Kletzel, S. L., Cary, M. P., Ciro, C., Berbrayer, D., Dawson, D., Hoffecker, L., ... y Heyn, P. C. (2016). Brain Gaming: A User's Product Guide for the Clinician. Archives of Physical Medicine and Rehabilitation, 97(8), 1399-1400. https://doi.org/10.1016/j.apmr.2016.03.001

22. Kueider, A. M., Parisi, J. M., Gross, A. L., y Rebok, G. W. (2012). Computerized cognitive training with older adults: a systematic review. PloS One, 7(7), e40588. https://doi.org/10.1371/journal.pone.00405

23. Love, S., Kannis-Dymand, L., y Lovell, G. P. (2018). Metacognitions in Triathletes: Associations With Attention, State Anxiety, and Relative Performance. Journal of Applied Sport 


\section{Validez convergente Rejilla informática para deportistas}

Psychology, 30(4),

421-436. https://doi.org/10.1080/10413200.2018.1440660

24. Memmert, D. (2009). Pay attention! A review of visual attentional expertise in sport. International Review of Sport and Exercise Psychology, 2(2), 119-138. https://doi.org/10.1080/17509840802641372

25. Morrison, G.E., Simone, C.M., Ng, N.F., Hardy, J.L. (2015). Reliability and validity of the NeuroCognitive Performance Test, a web-based neuropsychological assessment. Frontiers in Psychology, 6,1652 . https://doi.org/10.3389/fpsyg.2015.01652

26. O'halloran, L., Cao, Z., Ruddy, K., Jollans, L., Albaugh, M. D., Aleni, A., ... y Bokde, A. L. (2018). Neural circuitry underlying sustained attention in healthy adolescents and in ADHD symptomatology. Neuroimage, 169, 395-406. https://doi.org/10.1016/j.neuroimage.2017.12.030

27. Posner, M.I. y Petersen, S.E. (1990). The attention system of the human brain. Annual Review of Neuroscience, $13, \quad 25-42$. https://doi.org/10.1146/annurev.ne.13.030190.00 0325

28. Rabiner, D.L., Murray, D.W., Skinner, A.T. y Malone, P.S. (2010). A randomized trial of two promising computer-based interventions for students with attention difficulties. Journal of Abnormal Child Psychology, 38, 131-142. https://doi.org/10.1007/s10802-009-9353-x

29. Reigal, R. E., González-Guirval, F., Morillo Baro, J. P., Morales-Sánchez, V., Juárez-Ruiz de Mier, R., y Hernández-Mendo, A. (2019). Effects of a computerized training on attentional capacity of young soccer players. Frontiers in Psychology, 10 , 2279. http://dx.doi.org/10.3389/fpsyg.2019.02279

30. Roca, A., Ford, P. R., y Memmert, D. (2018). Creative decision making and visual search behavior in skilled soccer players. PloS One, 13(7), e0199381. https://doi.org/10.1371/journal.pone.0199381

31. Romeas, T., Guldner, A., y Faubert, J. (2016). 3DMultiple Object Tracking training task improves passing decision-making accuracy in soccer players. Psychology of Sport and Exercise, 22, 1-
9. https://doi.org/10.1016/j.psychsport.2015.06.002

32. Rosenberg, M. D., Finn, E. S., Scheinost, D., Papademetris, X., Shen, X., Constable, R. T., y Chun, M. M. (2016). A neuromarker of sustained attention from whole-brain functional connectivity. Nature Neuroscience, 19(1), 165171. http://dx.doi.org/10.1038/nn.4179

33. Rosenberg, M. D., Finn, E. S., Scheinost, D., Constable, R. T., y Chun, M. M. (2017). Characterizing attention with predictive network models. Trends in Cognitive Sciences, 21(4), 290302. https://doi.org/10.1016/j.tics.2017.01.011

34. Schack, T., Bertollo, M., Koester, D., Maycock, J., y Essig, K. (2014). Technological advancements in sport psychology. En A. G. Papaioannou y D. Hackfort (Eds.), Routledge Companion to Sport and Exercise Psychology (pp. 953-965), London: Routledge).

35. Shatil, E., Mikulecka, J., Bellotti, F., y Bureš, V. (2014). Novel television-based cognitive training improves working memory and executive function. PLoS One, 9(7), e101472. https://doi.org/10.1371/journal.pone.0101472

36. Stuss, D. T. (2011). Functions of the frontal lobes: relation to executive functions. Journal of the International Neuropsychological Society, 17(5), 759-765. https://doi.org/10.1017/S1355617711000695

37. Tamm, L., Epstein, J. N., Peugh, J. L., Nakonezny, P. A., y Hughes, C. W. (2013). Preliminary data suggesting the efficacy of attention training for school-aged children with ADHD. Developmental Cognitive Neuroscience, 4, 16-28. https://doi.org/10.1016/j.dcn.2012.11.004

38. Toulouse, E. y Piéron, H. (1992). Prueba perceptiva y de atención. Madrid: TEA Ediciones.

39. Walsh, J. J., Dunlap, C., Miranda, J., Thorp, D. B., Kimmerly, D. S., Tschakovsky, M., y Gurd, B. J. (2018). Brief, high-intensity interval exercise improves selective attention in university students. International Journal of Exercise Science, 11(5), 152-167.

40. Wass, S., Porayska-Pomsta, K. y Johnson, M.H. (2011). Training attentional control in infancy. 


\section{González-Guirval, Reigal, Morillo-Baro, Juárez-Ruiz de Mier, Hernández-Mendo, \& Morales- Sánchez}

Current Biology, 21(18), 1543-1547. https://doi.org/10.1016/j.cub.2011.08.004

41. Wilson, M. R., Vine, S. J., y Wood, G. (2009). The influence of anxiety on visual attentional control in basketball free throw shooting. Journal of Sport and Exercise Psychology, 31(2), 152-168. https://doi.org/10.1123/jsep.31.2.152

42. World Medical Association (2013). World Medical Association Declaration of Helsinki: Ethical principles for medical research involving human subjects. Journal of the American Medical Association, 310, 2191-2194. https://doi.org/10.1001/jama.2013.281053

43. Zaehringer, J., Falquez, R., Schubert, A. L., Nees, F., y Barnow, S. (2018). Neural correlates of reappraisal considering working memory capacity and cognitive flexibility. Brain Imaging and Behavior, 12(6), 1529-1543. https://doi.org/10.1007/s11682-017-9788-6 